\title{
POLYNOMIALS OVER FINITE FIELDS WHICH COMMUTE WITH LINEAR PERMUTATIONS
}

\author{
GARY L. MULLEN
}

\begin{abstract}
The polynomials over a finite field which commute with an arbitrary linear permutation of the field are characterized. This characterization is obtained through the use of a result from Pólya's theory of enumeration.
\end{abstract}

Let $p$ be a prime number, $q=p^{n}$ for some positive integer $n$, and $\operatorname{GF}(q)$ the finite field with $q$ elements. Let $a$ be a nonzero element of $\operatorname{GF}(q)$. In [3] Wells characterized those polynomials over $\operatorname{GF}(q)$ which commute with a translation by an element of the field, i.e., he characterized those polynomials $f(x)$ with coefficients in $\mathrm{GF}(q)$ for which $f(x+a)=f(x)+a$. In the present paper we characterize those polynomials $f(x)$ for which

$$
f(b x+a)=b f(x)+a
$$

where $b \in \mathrm{GF}(q)$. Thus if $b=1$, our characterization reduces to that considered by Wells.

Since, by the Lagrange Interpolation Formula [2, p. 55], every polynomial over $\mathrm{GF}(q)$ is congruent $\left(\bmod x^{q}-x\right)$ to a unique polynomial of degree $<q$, this will characterize all polynomials over $\operatorname{GF}(q)$ satisfying (1). The characterization will be obtained by equating coefficients in (1) and using an elementary result from Pólya's theory of enumeration.

Let $f(x)=b_{0}+b_{1} x+b_{2} x^{2} \ldots+b_{q-1} x^{q-1}$ where for $r=0,1, \ldots, q-1, b_{r}$ $\in \mathrm{GF}(q)$. As a simple special case suppose $b=0$ so that $f(x)$ satisfies (1) if and only if

$$
0=b_{0}+\left(b_{1}-1\right) a+b_{2} a^{2}+\cdots+b_{q-1} a^{q-1}
$$

and thus there are clearly $q^{q-1}$ polynomials with the property that $f(a)=a$. Suppose now that $b \neq 0$.

THEOREM 1. The polynomial $f(x)=b_{0}+b_{1} x+\cdots+b_{q-1} x^{q-1}$ satisfies (1) if and only if

$$
\begin{aligned}
& b_{0}(b-1)=-a+\sum_{t=1}^{q-1} b_{t} a^{t} \\
& b_{s}\left(1-b^{s-1}\right)=b^{s-1} \sum_{t=s+1}^{q-1}\left(\begin{array}{l}
t \\
s
\end{array}\right) b_{t} a^{t-s} \quad(1<s<q-1) .
\end{aligned}
$$

Received by the editors April 21, 1981 and, in revised form, May 29, 1981.

1980 Mathematics Subject Classification. Primary 12C05; Secondary 20B05.

Key words and phrases. Finite field, permutation. 
Proof. If $b=1$ the set of conditions (2) are both necessary and sufficient since they reduce to the set of conditions given by Wells in [3]. Hence we assume $b \neq 1$. If $c_{s}$ denotes the coefficient of $x^{s}$ in the polynomial $f(b x+a)$ then

$$
c_{s}=b^{s} \sum_{t=s}^{q-1}\left(\begin{array}{l}
t \\
s
\end{array}\right) b_{t} a^{t-s}
$$

so that if (1) is to hold, $c_{0}=b b_{0}+a$ and $c_{s}=b b_{s}$ for $1 \leqslant s \leqslant q-1$. Hence the conditions (2) are necessary if (1) is to hold.

To prove the sufficiency, suppose that the multiplicative order of $b$ is $k$ where $k \mid q-1$. Hence if $k \mid s-1$ then $b_{s}$ can be chosen arbitrarily from $\mathrm{GF}(q)$. If $k \nmid s-1$ then (2) determines $b_{s}$ uniquely in terms of $b_{s+1}, \ldots, b_{q-1}$. If $s=1$ then $b_{1}$ can be chosen arbitrarily and thus there are $[(q-2) / k]+1=(q-1) / k$ coefficients which can be chosen arbitrarily from $\operatorname{GF}(q)$. The remaining coefficients will be uniquely determined by (2). Hence there are $q^{(q-1) / k}$ polynomials $f(x)$ over $\operatorname{GF}(q)$ of degree $<q$ satisfying (2).

In [1] it is shown that if $g$ and $h$ are permutations of finite sets $D$ and $R$ where the types of $g$ and $h$ are $\left(d_{1}, d_{2}, \ldots\right)$ and $\left(e_{1}, e_{2}, \ldots\right)$, respectively, then the number of functions $f: D \rightarrow R$ for which $f g=h f$ is

$$
\prod_{i}\left(\sum_{j \mid i} j e_{j}\right)^{d_{i}} .
$$

We consider the case where $D=R=\mathrm{GF}(q)$ and $g=h=b x+a$ with $b \neq 0$. Since $b \neq 1$, it is not difficult to show that the polynomial $b x+a$ induces a permutation of $\operatorname{GF}(q)$ which is the product of $(q-1) / k$ disjoint $k$-cycles and one cycle of length one, the fixed point being $a /(1-b)$. Thus $d_{1}=1, d_{k}=(q-1) / k$ and $d_{i}=0$ if $i \neq 1$ or $k$ so that (3) reduces to $q^{(q-1) / k}$. By the Lagrange Interpolation Formula each function from $\operatorname{GF}(q)$ to itself is induced by exactly one polynomial of degree $<q$. Hence there are exactly $q^{(q-1) / k}$ polynomials $f(x)$ of degree $<q$ which satisfy (1) so that the conditions (2) are also sufficient. In addition we have proved

COROLlaRY 2. If $b \in \operatorname{GF}(q)$ has multiplicative order $k$ then the number of polynomials $f(x)$ of degree $<q$ with the property that $f(b x+a)=b f(x)+a$ is $q^{(q-1) / k}$.

Of course not all of the $q^{(q-1) / k}$ polynomials are permutations. By using another result from [1], it is easily seen that the number of $f$ which are 1-1 is given by

$$
\prod_{j} j^{d_{j}} e_{j}\left(e_{j}-1\right) \cdots\left(e_{j}-d_{j}+1\right)
$$

so that we can state

COROLlaRY 3. If $b \in \mathrm{GF}(q)$ has multiplicative order $k$ then the number of permutation polynomials $f(x)$ of degree $<q$ with the property that $f(b x+a)=b f(x)$ $+a$ is $k^{(q-1) / k}((q-1) / k)$ !. 
One can also easily obtain Wells' results by using (3) and (4). In particular, since $x+a$ induces a permutation which is the product of $p^{n-1}$ disjoint $p$-cycles, the number of polynomials $f(x)$ with the property that $f(x+a)=f(x)+a$ is $q^{p^{n-1}}$ while the number of such permutation polynomials is $p^{p^{n-1}}\left(p^{n-1}\right) !$.

Let $F_{m \times m}$ denote the algebra of $m \times m$ matrices over $F=\operatorname{GF}(q)$. If $b \neq 0$, $a \in \mathrm{GF}(q)$, then $\theta(x)=b x+a$ induces a permutation of $F_{m \times m}$ via substitution where if $D \in F_{m \times m}$ then $\theta(D)=b D+a I_{m}$ where $I_{m}$ is the $m \times m$ identity matrix. If $D=\left(d_{i j}\right), i=1, \ldots, m ; j=1, \ldots, m$, then the matrix $\theta(D)$ is determined by $\theta(D)=\left(b d_{i j}+a \delta_{i j}\right)$ where $\delta_{i j}$ is the Kronecker delta symbol. Hence if $b \neq 1$ has multiplicative order $k$ then $\theta(x)$ induces a permutation on $F_{m \times m}$ which is the product of $\left(q^{m^{2}}-1\right) / k$ disjoint $k$-cycles and one cycle of length one, the fixed matrix being $D=\left(d_{i j}\right)=\left(a \delta_{i j} /(1-b)\right)$. Proceeding as before we can prove

THEOREM 4. If $b \in \mathrm{GF}(q)$ has multiplicative order $k$ then the number of functions $f: F_{m \times m} \rightarrow F_{m \times m}$ with the property that

$$
f(b x+a)=b f(x)+a I_{m}
$$

is $\left(q^{m^{2}}\right)^{\left(q m^{2}-1\right) / k}$ of which $k^{\left(q m^{2}-1\right) / k}\left(\left(q^{m^{2}}-1\right) / k\right)$ ! are permutations of $F_{m \times m}$.

If $b=1$ then $\theta(x)$ induces a permutation on $F_{m \times m}$ which is the product of $q^{m^{2}} / p$ disjoint $p$-cycles so we can state

THEOREM 5. The number of functions $f: F_{m \times m} \rightarrow F_{m \times m}$ with the property that $f(x+a)=f(x)+a I_{m}$ is $\left(q^{m^{2}}\right)^{q^{m^{2}} / k}$ of which $p^{p^{m m^{2}-1}}\left(p^{n m^{2}-1}\right)$ ! are permutations of $F_{m \times m}$.

We note that if $m=1$ the results of Theorems 4 and 5 reduce to those of Corollaries 2 and 3 along with the results of Wells.

\section{REFERENCES}

1. N. G. deBruijn, Pólya's theory of counting, Applied Combinatorial Mathematics, (Ed., E. F. Beckenbach), Wiley, New York, 1964.

2. L. E. Dickson, Linear groups: With an exposition of the Galois field theory, Dover, New York, 1958.

3. C. Wells, Polynomials over finite fields which commute with translation, Proc. Amer. Math. Soc. 46 (1974), 347-350.

Department of Mathematics, Pennsylvania State University, Shenango Valley Campus, Sharon, Pennsylvania 16146 